\title{
Re: International System for Human Cytogenetic or Cytogenomic Nomenclature (ISCN): Some Thoughts, by T. Liehr
}

\author{
Jean McGowan-Jordan ${ }^{a}$ Ros Hastings ${ }^{b}$ Sarah Moore ${ }^{c}$ \\ aGenetics, CHEO/University of Ottawa, Ottawa, ON, Canada; bJohn Radcliffe Hospital, \\ Oxford University Hospitals NHS Foundation Trust, Oxford, UK; ' ${ }^{C}$ Genetics and Molecular Pathology, \\ SA Pathology, SA Genomics Health Alliance, Adelaide, SA, Australia
}

\section{Keywords}

International System for Human Cytogenomic Nomenclature (ISCN) · Cytogenomics · Band level · Idiograms

As members of the International System for Human Cytogenomic Nomenclature Standing Committee and editors of the recently published ISCN 2020, we wish to respond to the comments of Dr. Thomas Liehr.

First, over a decade ago, cytogenomics (molecular cytogenomics) was introduced to define a body of research in human genomics (genetics) focused on genomic variations and architecture at microscopic/submicroscopic level and at molecular resolutions [Iourov et al., 2008]. The adoption of cytogenomics to encompass cytogenetics and molecular techniques is to be encouraged, rather than discouraged, by traditionally trained cytogeneticists. For many referrals, cytogenetic analysis on its own has been replaced as a first-line genome-wide technique with more molecular-based techniques which are now the first-line test, e.g., microarrays and next-generation sequencing.
Second, the cover art, is just that: art. Its purpose is to distinguish, readily, the 2020 edition from earlier editions. Dr. Liehr's preference for a chromosome on the cover is noted, and the editor of the next edition will make an informed decision for the artwork for the next edition of the ISCN.

It is unfortunate that Dr. Liehr did not mention his concerns regarding the precision of the band levels depicted in the idiograms in the call for comments/improvements prior to the 2019 meeting of the Standing Committee, where all suggestions were considered. Renaming the banding resolution is not appropriate since the levels of resolution given provide only an estimate. The assertion that an estimate allows only \pm 5 error is rejected, although the editors of the next edition may consider renaming the 700 -band resolution as 750 bands assuming their review of the resolution confirms that this is required.

Finally, the overall statement that pathogenic/likely pathogenic/variant of unknown significance/likely benign/benign are for monogenic disorders and SNPs is not helpful. Indeed this classification is now used for arrays to determine whether the $\mathrm{CNV}$ is likely to be clinically relevant and is endorsed by various guidelines and databases

Correspondence to:

Jean McGowan-Jordan, jordan@ cheo.on.ca 
[e.g., Kearney et al., 2011]. Additionally, the number of pages that deal with cytogenetics is irrelevant; the title reflects the content of the book which is cytogenomics.

We thank Thomas Liehr for his comments on ISCN and hope our response clarifies some of the issues raised.

\section{Conflict of Interest Statement}

The authors have no conflicts of interest to declare.

\section{Funding Sources}

Not applicable.

\section{Author Contributions}

The authors contributed equally to this commentary.

\section{References}

Iourov IY, Vorsanova SG, Yurov YB. Molecular cytogenetics and cytogenomics of brain diseases. Curr Genomics. 2008;9:452-65.

Kearney HM, Thorland EC, Brown KK, Quintero-Rivera F, South ST. American College of Medical Genetics standards and guidelines for interpretation and reporting of postnatal constitutional copy number variants. Genet Med. 2011;13:680-5. 